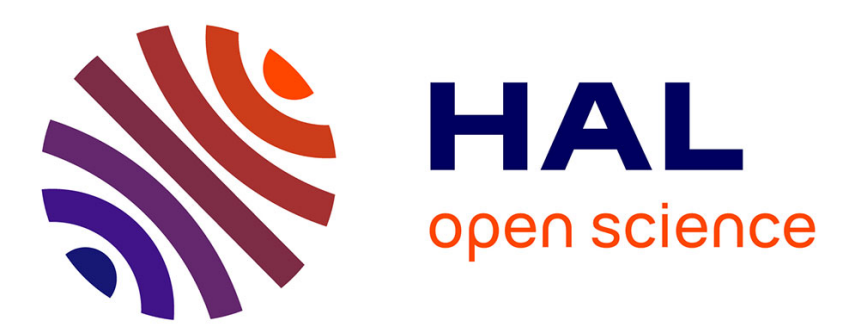

\title{
Metastability of reversible condensed zero range processes on a finite set
}

Johel Beltran, Claudio Landim

\section{To cite this version:}

Johel Beltran, Claudio Landim. Metastability of reversible condensed zero range processes on a finite set. 2009. hal-00425463

\section{HAL Id: hal-00425463 \\ https://hal.science/hal-00425463}

Preprint submitted on 21 Oct 2009

HAL is a multi-disciplinary open access archive for the deposit and dissemination of scientific research documents, whether they are published or not. The documents may come from teaching and research institutions in France or abroad, or from public or private research centers.
L'archive ouverte pluridisciplinaire HAL, est destinée au dépôt et à la diffusion de documents scientifiques de niveau recherche, publiés ou non, émanant des établissements d'enseignement et de recherche français ou étrangers, des laboratoires publics ou privés. 


\title{
METASTABILITY OF REVERSIBLE CONDENSED ZERO RANGE PROCESSES ON A FINITE SET
}

\author{
J. BELTRÁN, C. LANDIM
}

\begin{abstract}
Let $r: S \times S \rightarrow \mathbb{R}_{+}$be the jump rates of an irreducible random walk on a finite set $S$, reversible with respect to some probability measure $m$. For $\alpha>1$, let $g: \mathbb{N} \rightarrow \mathbb{R}_{+}$be given by $g(0)=0, g(1)=1, g(k)=(k / k-1)^{\alpha}$, $k \geq 2$. Consider a zero range process on $S$ in which a particle jumps from a site $x$, occupied by $k$ particles, to a site $y$ at rate $g(k) r(x, y)$. Let $N$ stand for the total number of particles. In the stationary state, as $N \uparrow \infty$, all particles but a finite number accumulate on one single site. We show in this article that in the time scale $N^{1+\alpha}$ the site which concentrates almost all particles evolves as a random walk on $S$ whose transition rates are proportional to the capacities of the underlying random walk.
\end{abstract}

\section{INTRODUCTION}

Fix a finite state space $S$ of cardinality $\kappa \geq 2$ and consider an irreducible continuous time random walk $\left\{X_{t}: t \geq 0\right\}$ on $S$ which jumps from $x$ to $y$ at some rate $r(x, y)$. Assume that this dynamics is reversible with respect to some probability measure $m$ on $S: m(x) r(x, y)=m(y) r(y, x), x, y \in S$. Denote by cap ${ }_{S}$ the capacity associated to this random walk: For two disjoint proper subsets $A, B$ of $S$,

$$
\operatorname{cap}_{S}(A, B)=\inf _{f \in \mathcal{B}(A, B)} \frac{1}{2} \sum_{x, y \in S} m(x) r(x, y)\{f(y)-f(x)\}^{2},
$$

where $\mathcal{B}(A, B)$ stands for the set of functions $f: S \rightarrow \mathbb{R}$ equal to 1 at $A$ and equal to 0 at $B$. When $A=\{x\}, B=\{y\}$, we represent $\operatorname{cap}_{S}(A, B)$ by $\operatorname{cap}_{S}(x, y)$.

Let $M_{\star}$ be the maximum value of the probability measure $m: M_{\star}=\max \{m(x)$ : $x \in S\}$ and denote by $S_{\star}$ the sites where $m$ attains its maximum value: $S_{\star}=$ $\left\{x \in S: m(x)=M_{\star}\right\}$. Of course, in the symmetric, nearest-neighbor case, where $r(x, y)=1$ if $y=x \pm 1$, modulo $\kappa$, and $r(x, y)=0$ otherwise, $m$ is constant and $S_{\star}$ and $S$ coincide.

Fix a real number $\alpha>1$. Let $g: \mathbb{N} \rightarrow \mathbb{R}$ be given by

$$
g(0)=0, \quad g(1)=1, \quad \text { and } \quad g(n)=\left(\frac{n}{n-1}\right)^{\alpha}, n \geq 2,
$$

so that $\prod_{i=1}^{n} g(i)=n^{\alpha}, n \geq 1$. Consider the zero range process on $S$ in which a particle jumps from a site $x$, occupied by $k$ particles, to a site $y$ at rate $g(k) r(x, y)$. Since $g$ is decreasing, the dynamics is attractive in the sense that particles on sites with a large number of particles leave them at a slower rate than particles on sites with a small number of particles.

The total number of particles is conserved by the dynamics, and for each fixed integer $N \geq 1$ the process restricted to the set of configurations with $N$ particles,

Key words and phrases. Metastability, condensation, zero range processes. 
denoted by $E_{N}$, is irreducible. Let $\mu_{N}$ be the unique invariant probability measure on $E_{N}$. When $\alpha>2$, the measure $\mu_{N}$ exhibits a very peculiar structure called condensation in the physics literature. Mathematically, this means that under the stationary state, above a certain critical density, as the total number of particles $N \uparrow \infty$, only a finite number of particles are located on the sites which do not contain the largest number of particles.

Condensation has been observed and investigated in shaken granular systems, growing and rewiring networks, traffic flows and wealth condensation in macroeconomics. We refer to the recent review by Evans and Hanney [4].

Several aspects of the condensation phenomenon for zero range dynamics have been examined. Let the condensate be the site with the maximal occupancy. Precise estimates on the number of particles at the condensated, as well as its fluctuations, have been obtained in $[9,8,5]$. The equivalence of ensembles has been proved by Großkinsky, Schütz and Spohn [8]. Ferrari, Landim and Sisko [6] proved that if the number of sites is kept fixed, as the total number of particles $N \uparrow \infty$, the distribution of particles outside the condensated converges to the grand canonical distribution with critical density. Armendariz and Loulakis [1] generalized this result showing that if the number of sites $\kappa$ grows with the number of particles $N$ in such a way that the density $N / \kappa$ converges to a value greater than the critical density, the distribution of the particles outside the condensate converges to the grand canonical distribution with critical density.

We investigate in this article the dynamical aspects of the condensation phenomenon. Fix an initial configuration with the majority of particles located at one site. Denote by $X_{t}^{N}$ the position of the condensated at time $t \geq 0$. In case of ties, $X_{t}^{N}$ remains in the last position. The process $\left\{X_{t}^{N}: t \geq 0\right\}$ evolves randomly on $S$ according to some non-Markovian dynamics.

The main result of this article states that, for $\alpha>1$, in the time scale $N^{1+\alpha}$, the process $\left\{X_{t}^{N}: t \geq 0\right\}$ evolves asymptotically according to a random walk on $S_{\star}$ which jumps from $x$ to $y$ at a rate proportional to the capacity $\operatorname{cap}_{S}(x, y)$. In the terminology of [2], we are proving that the condensate exhibits a tunneling behavior in the time scale $N^{1+\alpha}$.

This article leaves two interesting open questions. The techniques used here rely strongly on the reversibility of the process. It is quite natural to examine the same problem for asymmetric zero range processes where new techniques are required. On the other hand, the number of sites is kept fixed. It is quite tempting to let the number of sites grow with the number of particles. In this case, in the nearest neighbor, symmetric model, for instance, the condensate jumps from one site to another at rate proportional to the inverse of the distance. The rates are therefore not summable and it is not clear if a scaling limit exists.

Simulations for the evolution of the condensated have been performed by Godrèche and Luck [7]. The authors predicted the time scale, obtained here, in which the condensate evolves and claimed that the time scale should be the same for non reversible dynamics.

\section{Notation AND RESUlts}

Throughout this article we fix a finite set $S$ of cardinality $\kappa \geq 2$ and a real number $\alpha>1$. For each $S_{0} \subseteq S$ consider the set of configurations $E_{N, S_{0}}, N \geq 1$, 
given by

$$
E_{N, S_{0}}:=\left\{\eta \in \mathbb{N}^{S_{0}}: \sum_{x \in S_{0}} \eta_{x}=N\right\},
$$

where $\mathbb{N}=\{0,1,2, \ldots\}$. When $S_{0}=S$, we use the shorthand $E_{N}$ for $E_{N, S}$. Define $a(n)=n^{\alpha}$ for $n \geq 1$ and set $a(0)=1$. Let us also define $g: \mathbb{N} \rightarrow \mathbb{R}_{+}$,

$$
g(0)=0, \quad g(1)=1 \quad \text { and } \quad g(n)=\frac{a(n)}{a(n-1)}, \quad n \geq 2,
$$

in such a way that $\prod_{i=1}^{n} g(i)=a(n), n \geq 1$, and $\{g(n): n \geq 2\}$ is a strictly decreasing sequence converging to 1 as $n \uparrow \infty$.

Consider a random walk on $S$ with jump rates denoted by $r$. Its generator $\mathcal{L}_{S}$ acts on functions $f: S \rightarrow \mathbb{R}$ as

$$
\left(\mathcal{L}_{S} f\right)(x)=\sum_{y \in S} r(x, y)\{f(y)-f(x)\} .
$$

Assume that this Markov process is irreducible and reversible with respect to some probability measure $m$ on $S$ :

$$
m(x) r(x, y)=m(y) r(y, x), \quad x, y \in S .
$$

Let $M_{\star}$ be the maximum value of the probability measure $m$, let $S_{\star} \subset S$ be the sites of $S$ where $m$ attains its maximum and let $\kappa_{\star}$ be the cardinality of $S_{\star}$ :

$$
M_{\star}=\max \{m(x): x \in S\}, \quad S_{\star}=\left\{x \in S: m(x)=M_{\star}\right\} \quad \text { and } \quad \kappa_{\star}=\left|S_{\star}\right| .
$$

In addition, let $m_{\star}(x)=m(x) / M_{\star}$ so that $m_{\star}(x)=1$ for any $x \in S_{\star}$. Denote by $D_{S}$ the Dirichlet form associated to the random walk:

$$
D_{S}(f)=\frac{1}{2} \sum_{x, y \in S} m(x) r(x, y)\{f(y)-f(x)\}^{2}
$$

for $f: S \rightarrow \mathbb{R}$, and denote by $\operatorname{cap}_{S}(x, y)$ the capacity between two different points $x, y \in S:$

$$
\operatorname{cap}_{S}(x, y)=\inf _{f \in \mathcal{B}(x, y)}\left\{D_{S}(f)\right\},
$$

where the infimum is carried over the set $\mathcal{B}(x, y)$ of all functions $f: S \rightarrow \mathbb{R}$ such that $f(x)=1$ and $f(y)=0$.

For each pair $x, y \in S, x \neq y$, and $\eta \in E_{N}$ such that $\eta_{x}>0$, denote by $\sigma^{x y} \eta$ the configuration obtained from $\eta$ by moving a particle from $x$ to $y$ :

$$
\left(\sigma^{x y} \eta\right)_{z}= \begin{cases}\eta_{x}-1 & \text { for } z=x \\ \eta_{y}+1 & \text { for } z=y \\ \eta_{z} & \text { otherwise }\end{cases}
$$

For each $N \geq 1$, consider the zero range process defined as the Markov process $\left\{\eta^{N}(t): t \geq 0\right\}$ on $E_{N}$ whose generator $L_{N}$ acts on functions $F: E_{N} \rightarrow \mathbb{R}$ as

$$
\left(L_{N} F\right)(\eta)=\sum_{\substack{x, y \in S \\ x \neq y}} g\left(\eta_{x}\right) r(x, y)\left\{F\left(\sigma^{x y} \eta\right)-F(\eta)\right\} .
$$

The Markov process corresponding to $L_{N}, N \geq 1$, is irreducible and reversible with respect to its unique invariant measure $\mu_{N}$ given by

$$
\mu_{N}(\eta)=\frac{N^{\alpha}}{Z_{N, S}} \prod_{x \in S} \frac{m_{\star}(x)^{\eta_{x}}}{a\left(\eta_{x}\right)}:=\frac{N^{\alpha}}{Z_{N, S}} \frac{m_{\star}^{\eta}}{a(\eta)}, \quad \eta \in E_{N},
$$


where, for any $S_{0} \subseteq S$,

$$
m_{\star}^{\zeta}=\prod_{x \in S_{0}} m_{\star}(x)^{\zeta_{x}}, \quad a(\zeta)=\prod_{x \in S_{0}} a\left(\zeta_{x}\right), \quad \zeta \in \mathbb{N}^{S_{0}},
$$

and $Z_{N, S_{0}}$ is the normalizing constant

$$
Z_{N, S_{0}}=N^{\alpha} \sum_{\zeta \in E_{N, S_{0}}} \frac{m_{\star}^{\zeta}}{a(\zeta)} .
$$

In Section 3 we show that the sequence $\left\{Z_{N, S}: N \geq 1\right\}$ converges as $N \uparrow \infty$. This explains the factor $N^{\alpha}$ in its definition. The precise statement is as follows. For $x$ in $S$ and $\kappa \geq 2$, let

$$
\Gamma_{x}:=\sum_{j \geq 0} \frac{m_{\star}(x)^{j}}{a(j)}, \quad \Gamma(\alpha):=\sum_{j \geq 0} \frac{1}{a(j)}
$$

so that $\Gamma(\alpha)=\Gamma_{x}$ for any $x \in S_{\star}$, and define

$$
Z_{S}:=\frac{\kappa_{\star}}{\Gamma(\alpha)} \prod_{z \in S} \Gamma_{z}=\kappa_{\star} \Gamma(\alpha)^{\kappa_{\star}-1} \prod_{y \notin S_{\star}} \Gamma_{y} .
$$

Proposition 2.1. For every $\kappa \geq 2$,

$$
\lim _{N \rightarrow \infty} Z_{N, S}=Z_{S} .
$$

Denote by $D_{N}$ the Dirichlet form associated to the generator $L_{N}$. An elementary computation shows that

$$
D_{N}(F)=\frac{1}{2} \sum_{\substack{x, y \in S \\ x \neq y}} \sum_{\eta \in E_{N}} \mu_{N}(\eta) g\left(\eta_{x}\right) r(x, y)\left\{F\left(\sigma^{x y} \eta\right)-F(\eta)\right\}^{2},
$$

for every $F: E_{N} \rightarrow \mathbb{R}$.

For every two disjoint subsets $A, B$ of $E_{N}$ denote by $\mathcal{C}_{N}(A, B)$ the set of functions $F: E_{N} \rightarrow \mathbb{R}$ defined by

$$
\mathcal{C}_{N}(A, B):=\{F: F(\eta)=1 \forall \eta \in A \text { and } F(\xi)=0 \forall \xi \in B\} .
$$

The capacity corresponding to this pair of disjoint subsets $A, B$ is defined as

$$
\operatorname{cap}_{N}(A, B):=\inf \left\{D_{N}(F): F \in \mathcal{C}_{N}(A, B)\right\} .
$$

Since $D_{N}(F)=D_{N}(1-F), \operatorname{cap}_{N}(A, B)=\operatorname{cap}_{N}(B, A)$.

Fix a sequence $\left\{\ell_{N}: N \geq 1\right\}$ such that $1 \ll \ell_{N} \ll N$ and, for each $z \in S \backslash S_{\star}$, fix a sequence $\left\{b_{N}(z): N \geq 1\right\}$ such that $1 \ll b_{N}(z)$ :

$$
\lim _{N \rightarrow \infty} \ell_{N}=\infty, \quad \lim _{N \rightarrow \infty} \ell_{N} / N=0, \text { and } \lim _{N \rightarrow \infty} b_{N}(z)=\infty,
$$

for all $z \in S \backslash S_{\star}$. For $x$ in $S_{\star}$, let

$$
\mathcal{E}_{N}^{x}:=\left\{\eta \in E_{N}: \eta_{x} \geq N-\ell_{N}, \eta_{z} \leq b_{N}(z), z \notin S_{\star}\right\} .
$$

Obviously, $\mathcal{E}_{N}^{x} \neq \varnothing$ for all $x \in S_{\star}$ and every $N$ large enough. In the case where the measure $m$ is uniform, the second condition is meaningless and the set $\mathcal{E}_{N}^{x}$ becomes $\mathcal{E}_{N}^{x}=\left\{\eta \in E_{N}: \eta_{x} \geq N-\ell_{N}\right\}$.

Condition $\ell_{N} / N \rightarrow 0$ is required to guarantee that on each set $\mathcal{E}_{N}^{x}$ the proportion of particles at $x \in S_{\star}$, i.e. $\eta_{x} / N$, is almost one. As a consequence, for $N$ sufficiently 
large, the subsets $\mathcal{E}_{N}^{x}, x \in S_{\star}$, are pairwise disjoint. ¿From now on, we assume that $N$ is large enough so that the partition

$$
E_{N}=\left(\bigcup_{x \in S_{\star}} \mathcal{E}_{N}^{x}\right) \cup \Delta_{N}
$$

is well defined, where $\Delta_{N}$ is the set of configurations which do not belong to any set $\mathcal{E}_{N}^{x}, x \in S_{\star}$.

The assumptions that $\ell_{N} \uparrow \infty$ and that $b_{N}(z) \uparrow \infty$ for all $z \notin S_{\star}$ are sufficient to prove that $\mu_{N}\left(\Delta_{N}\right) \rightarrow 0$, as we shall see in (3.2), and to deduce the limit of the capacities stated in Theorem 2.2 below. In particular, in these two statements we may set $b_{N}(z)=N, z \notin S_{\star}$, in order to discard the second restriction in the definition of the sets $\mathcal{E}_{N}^{x}, x \in S_{\star}$. We need, however, further restrictions on the growth of $\ell_{N}$ and $b_{N}(z)$ to prove the tunneling behaviour of the zero range processes presented in Theorem 2.4 below.

To state the first main result of this article, for any nonempty subset $S_{\star}^{\prime}$ of $S_{\star}$, let $\mathcal{E}_{N}\left(S_{\star}^{\prime}\right)=\cup_{x \in S_{\star}^{\prime}} \mathcal{E}_{N}^{x}$, and let

$$
I_{\alpha}:=\int_{0}^{1} u^{\alpha}(1-u)^{\alpha} d u .
$$

Theorem 2.2. Assume that $\kappa_{\star} \geq 2$. Fix a nonempty subset $S_{\star}^{1} \subsetneq S_{\star}$ and denote $S_{\star}^{2}=S_{\star} \backslash S_{\star}^{1}$. Then,

$$
\lim _{N \rightarrow \infty} N^{1+\alpha} \operatorname{cap}_{N}\left(\mathcal{E}_{N}\left(S_{\star}^{1}\right), \mathcal{E}_{N}\left(S_{\star}^{2}\right)\right)=\frac{1}{M_{\star} \kappa_{\star} \Gamma(\alpha) I_{\alpha}} \sum_{x \in S_{\star}^{1}, y \in S_{\star}^{2}} \operatorname{cap}_{S}(x, y) .
$$

Note that the right hand side depends on the sites not in $S_{\star}$ through the capacities $\operatorname{cap}_{S}(x, y)$. In the case where the measure $m$ is constant, $S_{\star}=S, M_{\star}=\kappa_{\star}^{-1}$ and the right hand side becomes

$$
\frac{1}{\Gamma(\alpha) I_{\alpha}} \sum_{x \in S_{\star}^{1}, y \in S_{\star}^{2}} \operatorname{cap}_{S}(x, y) .
$$

To prove Theorem 2.2, we derive a lower and an upper bound for the capacity. In the first part, we need to obtain a lower bound for the Dirichlet form of functions in $\mathcal{C}_{N}\left(\mathcal{E}_{N}\left(S_{\star}^{1}\right), \mathcal{E}_{N}\left(S_{\star}^{2}\right)\right)$. To our advantage, since it is a lower bound, we may neglect some bonds in the Dirichlet form we believe to be irrelevant. On the other hand, and this is the main difficulty, the estimate must be uniform over $\mathcal{C}_{N}\left(\mathcal{E}_{N}\left(S_{\star}^{1}\right), \mathcal{E}_{N}\left(S_{\star}^{2}\right)\right)$. As we shall see in Section 4, the proof of a sharp lower bound gives a clear indication of the qualitative behavior of the function which solves the variational problem appearing in the definition of the capacity. With this information, we may propose a candidate for the upper bound. Here, in contrast with the first part, we have to estimate the Dirichlet form of a specific function, our elected candidate, but we need to estimate all the Dirichlet form and can not neglect any bond.

For each $\eta \in E_{N}$, let $\mathbf{P}_{\eta}^{N}$ stand for the probability on the path space $D\left(\mathbb{R}_{+}, E_{N}\right)$ induced by the zero range process $\left\{\eta^{N}(t): t \geq 0\right\}$ introduced in (2.4) starting from $\eta \in E_{N}$. Expectation with respect to $\mathbf{P}_{\eta}^{N}$ is denoted by $\mathbf{E}_{\eta}^{N}$. In addition, for any $A \subseteq E_{N}$, let $T_{A}$ denote the hitting time of $A$ :

$$
T_{A}:=\inf \left\{t \geq 0: \eta^{N}(t) \in A\right\} .
$$


Remark 2.3. It is well known (see e.g. Lemma 6.4 in [2]) that the solution of the variational problem for the capacity is given by

$$
\mathbf{F}_{\mathbf{S}_{\star}^{1}, \mathbf{S}_{\star}^{2}}(\eta)=\mathbf{P}_{\eta}^{N}\left[T_{\mathcal{E}_{N}\left(S_{\star}^{1}\right)}<T_{\mathcal{E}_{N}\left(S_{\star}^{2}\right)}\right] .
$$

The candidate proposed in the proof of the upper bound provides, therefore, an approximation, in the Dirichlet sense, of the function $\mathbf{F}_{\mathbf{S}_{\star}^{1}, \mathbf{S}_{\star}^{2}}$.

The second main result of this article states that the zero range process exhibits a metastable behavior as defined in [2]. Fix a nonempty subset $A$ of $E_{N}$. For each $t \geq 0$, let $\mathcal{T}_{t}^{A}$ be the time spent by the zero range process $\left\{\eta^{N}(t): t \geq 0\right\}$ on the set $A$ in the time interval $[0, t]$ :

$$
\mathcal{T}_{t}^{A}:=\int_{0}^{t} \mathbf{1}\left\{\eta^{N}(s) \in A\right\} d s
$$

and let $\mathcal{S}_{t}^{A}$ be the generalized inverse of $\mathcal{T}_{t}^{A}$ :

$$
\mathcal{S}_{t}^{A}:=\sup \left\{s \geq 0: \mathcal{T}_{s}^{A} \leq t\right\} .
$$

It is well known that the process $\left\{\eta^{N, A}(t): t \geq 0\right\}$ defined by $\eta^{N, A}(t)=\eta^{N}\left(\mathcal{S}_{t}^{A}\right)$ is a strong Markov process with state space $A$ [2]. This Markov process is called the trace of the Markov process $\left\{\eta^{N}(t): t \geq 0\right\}$ on $A$.

Consider the trace of $\left\{\eta^{N}(t): t \geq 0\right\}$ on $\mathcal{E}_{N}\left(S_{\star}\right)$, referred to as $\eta^{\mathcal{E}_{N}^{\star}}(t)$. Let $\Psi_{N}: \mathcal{E}_{N}\left(S_{\star}\right) \mapsto S_{\star}$ be given by

$$
\Psi_{N}(\eta)=\sum_{x \in S_{\star}} x \mathbf{1}\left\{\eta \in \mathcal{E}_{N}^{x}\right\}
$$

and let $X_{t}^{N}:=\Psi_{N}\left(\eta^{\varepsilon_{N}^{\star}}(t)\right)$.

We prove in Theorem 2.4 below that the speeded up non-Markovian process $\left\{X_{t N^{\alpha+1}}^{N}: t \geq 0\right\}$ converges to the random walk $\left\{X_{t}: t \geq 0\right\}$ on $S_{\star}$ whose generator $\mathbb{L}_{S_{\star}}$ is given by

$$
\left(\mathbb{L}_{S_{\star}} f\right)(x)=\frac{1}{M_{\star} \Gamma(\alpha) I_{\alpha}} \sum_{y \in S_{\star}} \operatorname{cap}_{S}(x, y)\{f(y)-f(x)\} .
$$

For $x$ in $S_{\star}$, denote by $\mathbb{P}_{x}$ the probability measure on the path space $D\left(\mathbb{R}_{+}, S_{\star}\right)$ induced by the random walk $\left\{X_{t}: t \geq 0\right\}$ starting from $x$.

Theorem 2.4. Assume that $\kappa_{\star} \geq 2$. If (2.6) holds and

$$
\lim _{N \rightarrow \infty} \frac{\ell_{N}^{1+\alpha(\kappa-1)}}{N^{1+\alpha}} \prod_{z \in S \backslash S_{\star}} m_{\star}(z)^{-b_{N}(z)}=0
$$

then, for each $x \in S_{\star}$,

(M1) We have

$$
\lim _{N \rightarrow \infty} \inf _{\eta, \xi \in \mathcal{E}_{N}^{x}} \mathbf{P}_{\eta}^{N}\left[T_{\{\xi\}}<T_{\mathcal{E}_{N}\left(S_{\star} \backslash\{x\}\right)}\right]=1 ;
$$

(M2) For any sequence $\xi_{N} \in \mathcal{E}_{N}^{x}, N \geq 1$, the law of the stochastic process $\left\{X_{t N^{\alpha+1}}^{N}: t \geq 0\right\}$ under $\mathbf{P}_{\xi_{N}}^{N}$ converges to $\mathbb{P}_{x}$ as $N \uparrow \infty$;

(M3) For every $T>0$,

$$
\lim _{N \rightarrow \infty} \sup _{\eta \in \mathcal{E}_{N}^{x}} \mathbf{E}_{\eta}^{N}\left[\int_{0}^{T} \mathbf{1}\left\{\eta^{N}\left(s N^{\alpha+1}\right) \in \Delta_{N}\right\} d s\right]=0 .
$$


If $\kappa>\kappa_{\star}$, in order to fulfill conditions (2.6) and (2.10), we can take, for instance,

$$
b_{N}(z)=\frac{-\log \left(\ell_{N}\right)}{\log \left(m_{\star}(z)\right)} \quad \text { for } z \in S \backslash S_{\star} \quad \text { and } \quad \ell_{N}=N^{1 /(\kappa-1)}
$$

if $\kappa_{\star} \geq 3, \ell_{N}=N^{1 /[\kappa-(1 / 2)]}$ if $\kappa_{\star}=2$.

According to the terminology introduced in [2], Theorem 2.4 states that the sequence of zero range processes $\left\{\eta^{N}(t): t \geq 0\right\}$ exhibits a tunneling behaviour on the time-scale $N^{\alpha+1}$ with metastates given by $\left\{\mathcal{E}_{N}^{x}: x \in S_{\star}\right\}$ and limit given by the random walk $\left\{X_{t}: t \geq 0\right\}$.

Property (M3) states that, outside a time set of order smaller than $N^{\alpha+1}$, one of the sites in $S_{\star}$ is occupied by at least $N-\ell_{N}$ particles. Property (M2) describes the time-evolution on the scale $N^{\alpha+1}$ of the site concentrating the largest number of particles. It evolves asymptotically as a Markov process on $S_{\star}$ which jumps from a site $x$ to $y$ at a rate proportional to the capacity $\operatorname{cap}_{S}(x, y)$ of the underlying random walk. Property (M1) guarantees that the process starting in a metastate $\mathcal{E}_{N}^{x}$ thermalizes therein before reaching any other metastate.

Remark 2.5. In [8], it is shown that, in the case the number of sites increases with the number of particles, the highest occupied site contains a nonzero fraction of the particles in the system. This result includes the case $1<\alpha \leq 2$. In contrast, when the number of sites is kept fixed, it seems to have been unnoticed in the literature that the condensation phenomenon appears also for $1<\alpha \leq 2$. More precisely, if $1 \ll \ell_{N} \ll N$, then

$$
\lim _{N \rightarrow \infty} \mu_{N}\left(\eta_{x} \geq N-\ell_{N}\right)=1 / \kappa_{\star}, \quad \forall x \in S_{\star} .
$$

Moreover, given that particles concentrate on $x \in S_{\star}$, the distribution of the configuration on $S \backslash\{x\}$ is asymptotically given by the grand-canonical measure determined by $m_{\star}$ : For any $x$ in $S_{\star}$,

$$
\lim _{N \rightarrow \infty} \sup _{\zeta \in \mathcal{G}_{N}^{x}}\left|\mu_{N}\left(\eta_{z}=\zeta_{z}, z \neq x \mid \eta_{x} \geq N-\ell_{N}\right)-\prod_{z \neq x} \frac{1}{\Gamma_{z}} \frac{m_{\star}(z)^{\zeta_{z}}}{a\left(\zeta_{z}\right)}\right|=0,
$$

where $\mathcal{G}_{N}^{x}:=\left\{\zeta \in \mathbb{N}^{S \backslash\{x\}}: \sum_{z} \zeta_{z} \leq \ell_{N}\right\}$. There is just a small difference between the cases $1<\alpha \leq 2$ and $\alpha>2$. While in the former, the variables $\left\{\eta_{z}: z \in S_{\star}\right\}$ do not have finite expectation under the critical grand-canonical measure, they do have finite expectation in the latter case.

In [3], we have proved Theorems 2.2 and 2.4 in the case where the rates $r(\cdot, \cdot)$ in the definition of the sequence of zero range processes corresponds to a random walk on a finite complete graph. Since it covers the case $\kappa=2$, we may suppose that $\kappa \geq 3$.

\section{The Stationary Measure $\mu_{N}$}

In this section, we prove Proposition 2.1. The proof relies on four lemmata. We first show that the sequence $Z_{N, S}$ is bounded below by a strictly positive constant and above by a finite constant. Let

$$
\tilde{Z}_{N, \kappa}=N^{\alpha} \sum_{\eta \in E_{N}} \frac{1}{a(\eta)}
$$

and note that $Z_{N, S} \leq \tilde{Z}_{N,|S|}$. 
Lemma 3.1. For each $\kappa \geq 2$, there exists a constant $A_{\kappa}>0$, which only depends on $\alpha$ and $\kappa$, such that

$$
1 \leq Z_{N, S} \leq \tilde{Z}_{N, \kappa} \leq A_{\kappa} .
$$

Proof. Choose $x$ in $S_{\star}$ and denote by $\xi$ the configuration in $E_{N}$ such that $\xi(x)=N$, $\xi(y)=0$ for $y \neq x$. By definition, $m_{\star}(x)=1$ so that $Z_{N, S} \geq N^{\alpha} m_{\star}^{\xi} / a(\xi)=1$, which proves the lower bound.

We proceed by induction to prove the upper bound. The estimate clearly holds for $\kappa=2$. Assume that it is in force for $2 \leq \kappa<k$. The identity

$$
\tilde{Z}_{N, k}=N^{\alpha}\left\{\frac{1}{N^{\alpha}}+\sum_{j=0}^{N-1} \frac{\tilde{Z}_{N-j, k-1}}{a(j) a(N-j)}\right\}
$$

permits to extend it to $\kappa=k$.

For any $\ell \geq 1$, let $E_{N, S}(\ell)$ be the subset of $E_{N, S}$ of all configurations with at most $N-\ell$ particles per site:

$$
E_{N, S}(\ell)=\left\{\eta \in E_{N, S}: \eta_{x} \leq N-\ell, \forall x \in S\right\} .
$$

Next lemma shows that the measure $\mu_{N}$ is concentrated on configurations in which all particles but a finite number accumulate at one site.

Lemma 3.2. There exists a constant $C_{\kappa}>0$ which only depends on $\alpha$ and $\kappa$, such that for every integer $\ell>0$,

$$
\sup _{N>\ell}\left\{N^{\alpha} \sum_{\eta \in E_{N, S}(\ell)} \frac{1}{a(\eta)}\right\} \leq \frac{C_{\kappa}}{\ell^{\alpha-1}} .
$$

Proof. We proceed by induction on $\kappa$. For $\kappa=2$ the statement is easily checked. Now, suppose the claim holds for $2 \leq \kappa \leq k-1$. Fix some $x$ in $S$. The left hand side of the inequality in the statement can be written as

$$
\sum_{\eta \in E_{N, S}(\ell)} \frac{N^{\alpha}}{a\left(\eta_{x}\right) a\left(N-\eta_{x}\right)} \frac{\left(N-\eta_{x}\right)^{\alpha}}{\prod_{y \neq x} a\left(\eta_{y}\right)} .
$$

This sum is equal to

$$
\left\{\sum_{0 \leq i \leq \ell / 2}+\sum_{\ell / 2<i \leq N-\ell}\right\} \frac{N^{\alpha}}{a(i) a(N-i)} \sum_{\xi \in E_{N-i, S \backslash\{x\}}(\ell-i)} \frac{(N-i)^{\alpha}}{a(\xi)},
$$

where the second sum is equal to zero if $\{i: \ell / 2<i \leq N-\ell\}$ is empty. We examine the two terms of this expression separately. By the induction assumption, the first sum is bounded above by

$$
\sum_{i=0}^{\ell / 2} \frac{N^{\alpha}}{a(i) a(N-i)} \frac{C_{k-1}}{(\ell-i)^{\alpha-1}} .
$$

By the previous lemma, this sum is less than or equal to

$$
\frac{2^{\alpha-1} C_{k-1}}{\ell^{\alpha-1}} \sum_{i=0}^{\ell / 2} \frac{N^{\alpha}}{a(i) a(N-i)} \leq \frac{2^{\alpha-1} C_{k-1} \tilde{Z}_{N, 2}}{\ell^{\alpha-1}} \leq \frac{2^{\alpha-1} C_{k-1} A_{2}}{\ell^{\alpha-1}} .
$$


On the other hand, by Lemma 3.1 and the induction assumption for $\kappa=2$, the second term in (3.1) is less than or equal to

$$
\sum_{\ell / 2<i \leq N-\ell} \frac{N^{\alpha}}{a(i) a(N-i)} \tilde{Z}_{N-i, k-1} \leq A_{k-1} C_{2}(2 / \ell)^{\alpha-1} .
$$

This concludes the proof of the lemma.

For $N \geq 2,0 \leq \ell \leq N, x \in S$, denote by $E_{N}^{x, \ell}$ the set of configurations in $E_{N, S}$ with at least $N-\ell$ particles at site $x$ :

$$
E_{N}^{x, \ell}=\left\{\eta \in E_{N, S}: \eta(x) \geq N-\ell\right\} .
$$

Recall the definition of the set $S_{\star}$. Next lemma shows that the $\mu_{N}-$ measure of the set $E_{N}^{x, \ell}$ decays exponentially if $x$ does not belong to $S_{\star}$.

Lemma 3.3. For each $\kappa \geq 2$, there exists a finite constant $C_{\kappa}$, depending only on $\kappa$ and $\alpha$, such that

$$
N^{\alpha} \sum_{\eta \in E_{N}^{x, \ell}} \frac{m_{\star}^{\eta}}{a(\eta)} \leq C_{\kappa} m_{\star}(x)^{N-\ell}
$$

for all $N>\ell$.

Proof. Fix $\kappa \geq 2$ and $x$ in $S$. The expression on the left hand side of the statement of the lemma is bounded by

$$
m_{\star}(x)^{N}+N^{\alpha} \sum_{i=N-\ell}^{N-1} \frac{m_{\star}(x)^{i}}{i^{\alpha}} \sum_{\xi \in E_{N-i, S \backslash\{x\}}} \frac{1}{a(\xi)} .
$$

By Lemma 3.1 and since $m_{\star}(x) \leq 1$, the second term is less than or equal to

$$
N^{\alpha} \sum_{i=N-\ell}^{N-1} \frac{m_{\star}(x)^{i}}{i^{\alpha}(N-i)^{\alpha}} \tilde{Z}_{N-i, \kappa-1} \leq A_{2} A_{\kappa-1} m_{\star}(x)^{N-\ell},
$$

which concludes the proof of the lemma.

If $\ell<N / 2$, the sets $\left\{E_{N}^{x, \ell}: x \in S\right\}$ are pairwise disjoint and

$$
E_{N, S} \backslash \bigcup_{x \in S} E_{N}^{x, \ell}=E_{N, S}(\ell+1) .
$$

It follows from the two previous lemmata that the sum in the definition of $Z_{N, S}$ restricted to configurations on $E_{N}^{x, \ell}, x \in S_{\star}$, is close to $\kappa_{\star}^{-1} Z_{N, S}$ for $\ell$ (and consequently $N$ ) large.

Lemma 3.4. For each $\kappa \geq 2$, there exists a constant $C_{\kappa}>0$, which only depends on $\alpha$ and $\kappa$, such that for every integer $\ell>0$ and $x \in S_{\star}$,

$$
\sup _{N>2 \ell}\left|N^{\alpha} \sum_{\eta \in E_{N}^{x, \ell}} \frac{m_{\star}^{\eta}}{a(\eta)}-\frac{Z_{N, S}}{\kappa_{\star}}\right| \leq \frac{C_{\kappa}}{\ell^{\alpha-1}} .
$$

Proof. As we have observed, for $0<\ell<N / 2$,

$$
Z_{N, S}=N^{\alpha} \sum_{x \in S} \sum_{\eta \in E_{N}^{x, \ell}} \frac{m_{\star}^{\eta}}{a(\eta)}+N^{\alpha} \sum_{\eta \in E_{N, S}(\ell+1)} \frac{m_{\star}^{\eta}}{a(\eta)} .
$$


By symmetry, for $x, y$ in $S_{\star}$,

$$
\sum_{\eta \in E_{N}^{x, \ell}} \frac{m_{\star}^{\eta}}{a(\eta)}=\sum_{\eta \in E_{N}^{y, \ell}} \frac{m_{\star}^{\eta}}{a(\eta)} .
$$

Hence, if $x$ belongs to $S_{\star}$,

$$
Z_{N, S}=\kappa_{\star} N^{\alpha} \sum_{\eta \in E_{N}^{x, \ell}} \frac{m_{\star}^{\eta}}{a(\eta)}+N^{\alpha} \sum_{y \notin S_{\star}} \sum_{\eta \in E_{N}^{y, \ell}} \frac{m_{\star}^{\eta}}{a(\eta)}+N^{\alpha} \sum_{\eta \in E_{N, S}(\ell+1)} \frac{m_{\star}^{\eta}}{a(\eta)} .
$$

The statement now follows from the two previous lemmata.

We are now in a position to prove the main result of this section.

Proof of Proposition 2.1. Fix a site $x$ in $S_{\star}$. By the previous lemma,

$$
\lim _{N \rightarrow \infty} \kappa_{\star}^{-1} Z_{N, S}=\lim _{\ell \rightarrow \infty} \lim _{N \rightarrow \infty} N^{\alpha} \sum_{\eta \in E_{N}^{x, \ell}} \frac{m_{\star}^{\eta}}{a(\eta)} .
$$

Since $x$ belongs to $S_{\star}$, the previous sum is equal to

$$
\sum_{j=0}^{\ell} \frac{N^{\alpha}}{(N-j)^{\alpha}} \sum_{\xi \in E_{j, S \backslash\{x\}}} \frac{m_{\star}^{\xi}}{a(\xi)}
$$

As $N \uparrow \infty$ and $\ell \uparrow \infty$, this expression converges to

$$
\sum_{j \geq 0} \sum_{\xi \in E_{j, S \backslash\{x\}}} \frac{m_{\star}^{\xi}}{a(\xi)}=\prod_{y \neq x} \sum_{j \geq 0} \frac{m_{\star}(y)^{j}}{a(j)}=\prod_{y \neq x} \Gamma_{y} .
$$

This concludes the proof of the proposition.

We close this section showing that

$$
\lim _{N \rightarrow \infty} \mu_{N}\left(\Delta_{N}\right)=0
$$

Recall the definition of the set $S_{\star}$ and of the sets $\mathcal{E}_{N}^{x}, x \in S_{\star}$. Since

$$
\Delta_{N}=\left[\bigcup_{z \in S \backslash S_{\star}}\left\{\eta: \eta_{z}>b_{N}(z)\right\}\right] \bigcup\left[\bigcap_{x \in S_{\star}}\left\{\eta: \eta_{x}<N-\ell_{N}\right\}\right],
$$

intersecting the second set with the partition $A=\cap_{z \in S \backslash S_{\star}}\left\{\eta: \eta_{z}<N-\ell_{N}\right\}$ and $A^{c}$, we get that

$$
\Delta_{N} \subset \bigcup_{z \in S \backslash S_{\star}} E_{N}^{z, c_{N}} \bigcup E_{N, S}\left(\ell_{N}+1\right),
$$

where $c_{N}=\min \left\{\ell_{N}, N-b_{N}(z): z \in S \backslash S_{\star}\right\}$. Hence, assertion (3.2) follows from Lemma 3.2, assumption (2.6) and Lemma 3.3. 


\section{LOWER BOUND}

In this section we prove a lower bound for the capacity. It might be simpler in a first reading to assume that $m$ is constant so that $S=S_{\star}$.

For $b, \ell \geq 3$ and $x, y$ in $S_{\star}, x \neq y$, consider the tube $L_{N}^{x, y}$ defined by

$$
L_{N}^{x, y}=\left\{\eta \in E_{N}: \eta_{x}+\eta_{y} \geq N-\ell ; \eta_{z} \leq b, z \in S \backslash S_{\star}\right\} .
$$

Clearly, $L_{N}^{x, y}=L_{N}^{y, x}$ for any $x, y \in S_{\star}$. We claim that for each $x \in S_{\star}$ and every $N$ sufficiently large

$$
L_{N}^{x, y} \cap L_{N}^{x, z} \subset \mathcal{E}_{N}^{x}, \quad y, z \in S_{\star} \backslash\{x\} .
$$

Indeed, let $\eta \in L_{N}^{x, y} \cap L_{N}^{x, z}$. First, $b \leq \inf _{z \in S \backslash S_{\star}} b_{N}(z)$ for any $N$ sufficiently large in view of (2.6). On the other hand, $\eta_{z} \leq \ell$ because $\eta$ belongs to $L_{N}^{x, y}$. Hence, $\eta_{x} \geq N-2 \ell$ since $\eta$ belongs to $L_{N}^{x, z}$. Since $\ell_{N} \rightarrow \infty$, this shows that $\eta_{x} \geq N-\ell_{N}$, for $N$ large enough and we conclude that $\eta \in \mathcal{E}_{N}^{x}$. Moreover, it follows from this argument that, for $N$ sufficiently large,

$$
L_{N}^{x, y} \cap L_{N}^{z, w} \neq \varnothing \quad \text { if and only if } \quad\{x, y\} \cap\{z, w\} \neq \varnothing .
$$

Proposition 4.1. Assume that $\kappa_{\star} \geq 2$. Fix a nonempty subset $S_{\star}^{1} \subsetneq S_{\star}$ and denote $S_{\star}^{2}=S_{\star} \backslash S_{\star}^{1}$. Then,

$$
\liminf _{N \rightarrow \infty} N^{1+\alpha} \operatorname{cap}_{N}\left(\mathcal{E}_{N}\left(S_{\star}^{1}\right), \mathcal{E}_{N}\left(S_{\star}^{2}\right)\right) \geq \frac{1}{M_{\star} \kappa_{\star} \Gamma(\alpha) I_{\alpha}} \sum_{x \in S_{\star}^{1}, y \in S_{\star}^{2}} \operatorname{cap}_{S}(x, y) .
$$

Proof. Fix a function $F$ in $\mathcal{C}_{N}\left(\mathcal{E}_{N}\left(S_{\star}^{1}\right), \mathcal{E}_{N}\left(S_{\star}^{2}\right)\right)$. By definition,

$$
D_{N}(F)=\frac{1}{2} \sum_{z, w \in S} \sum_{\eta \in E_{N}} \mu_{N}(\eta) r(z, w) g\left(\eta_{z}\right)\left\{F\left(\sigma^{z w} \eta\right)-F(\eta)\right\}^{2}
$$

We may bound from below the Dirichlet form $D_{N}(F)$ by

$$
\frac{1}{2} \sum_{x \in S_{\star}^{1}} \sum_{y \in S_{\star}^{2}} \sum_{z, w \in S} \sum_{\eta \in L_{N}^{x, y}} \mu_{N}(\eta) r(z, w) g\left(\eta_{z}\right)\left\{F\left(\sigma^{z w} \eta\right)-F(\eta)\right\}^{2} .
$$

In this inequality, we are neglecting several terms corresponding to configurations $\eta$ which do not belong to $\cup_{x \in S_{\star}^{1}, y \in S_{\star}^{2}} L_{N}^{x, y}$. On the other hand, some configurations are counted more than once because the sets $\left\{L_{N}^{x, y}: x \in S_{\star}^{1}, y \in S_{\star}^{2}\right\}$ are not disjoints. However, by (4.2), if $L_{N}^{x, y}$ and $L_{N}^{x^{\prime}, y^{\prime}}$ are different strips and $\eta$ belongs to $L_{N}^{x, y} \cap L_{N}^{x^{\prime}, y^{\prime}}$ then $x=x^{\prime}$ and $y \neq y^{\prime}$ (recall that $L_{N}^{x, y}=L_{N}^{y, x}$ ). In consequence, $\eta_{x} \geq N-2 \ell$. In particular, for $N$ large enough, $\eta$ and $\sigma^{z w} \eta$ belong to $\mathcal{E}_{N}^{x}$ for all $z, w \in S$, so that $F\left(\sigma^{z w} \eta\right)=F(\eta)$ because $F$ is constant on $\mathcal{E}_{N}^{x}$.

The proof of the lower bound has two steps. We first use the underlying random walk to estimate the Dirichlet form $D_{N}(F)$ by the capacity of this random walk multiplied by the Dirichlet form of a zero range process on two sites. This remaining Dirichlet form is easily bounded by explicit computations.

Fix $x \in S_{\star}^{1}, y \in S_{\star}^{2}$. Denote by $\mathfrak{d}_{x}, x \in S$, the configuration with one and only one particle at $x$, and agree that summation of configurations is performed 
componentwise. The change of variables $\xi=\eta-\mathfrak{d}_{z}$ shows that

$$
\begin{aligned}
& \frac{1}{2} \sum_{z, w \in S} \sum_{\eta \in L_{N}^{x, y}} \mu_{N}(\eta) r(z, w) g\left(\eta_{z}\right)\left\{F\left(\sigma^{z w} \eta\right)-F(\eta)\right\}^{2} \\
& \quad=\frac{1}{2} \sum_{z, w \in S} \sum_{\substack{\xi \in E_{N-1} \\
\xi+\mathfrak{d}_{z} \in L_{N}^{x, y}}} \frac{N^{\alpha}}{Z_{N, S}} \frac{m_{\star}^{\xi}}{a(\xi)} m_{\star}(z) r(z, w)\left\{F\left(\xi+\mathfrak{d}_{w}\right)-F\left(\xi+\mathfrak{d}_{z}\right)\right\}^{2} .
\end{aligned}
$$

This sum is clearly bounded below by

$$
\frac{1}{2} \sum_{z, w \in S} \sum_{\substack{\xi \in E_{N}-1 \\ \xi_{x}+\xi_{y} \geq N-\ell \\ \xi_{z} \leq b-1, \forall z \in S \backslash S_{\star}}} \frac{N^{\alpha}}{Z_{N, S}} \frac{m_{\star}^{\xi}}{a(\xi)} m_{\star}(z) r(z, w)\left\{F\left(\xi+\mathfrak{d}_{w}\right)-F\left(\xi+\mathfrak{d}_{z}\right)\right\}^{2} .
$$

Fix a configuration $\xi$ in $E_{N-1}$ and consider the function $f: S \rightarrow \mathbb{R}$ given by $f(v)=\left\{F\left(\xi+\mathfrak{d}_{v}\right)-F\left(\xi+\mathfrak{d}_{y}\right)\right\} /\left\{F\left(\xi+\mathfrak{d}_{x}\right)-F\left(\xi+\mathfrak{d}_{y}\right)\right\}$. Note that $f(x)=1$, $f(y)=0$. Moreover, if we recall the expression (2.2) of the Dirichlet form of the underlying random walk,

$$
\begin{aligned}
\frac{1}{2} \sum_{z, w \in S} m_{\star}(z) r(z, w)\left\{F\left(\xi+\mathfrak{d}_{w}\right)-F\left(\xi+\mathfrak{d}_{z}\right)\right\}^{2} \\
=\frac{1}{M_{\star}} D_{S}(f)\left\{F\left(\xi+\mathfrak{d}_{x}\right)-F\left(\xi+\mathfrak{d}_{y}\right)\right\}^{2}
\end{aligned}
$$

Since $f(x)=1, f(y)=0$, the previous expression is bounded below by

$$
\frac{1}{M_{\star}} \operatorname{cap}_{S}(x, y)\left\{F\left(\xi+\mathfrak{d}_{x}\right)-F\left(\xi+\mathfrak{d}_{y}\right)\right\}^{2} .
$$

Up to this point we proved that the Dirichlet form of $F$ is bounded below by

$$
\frac{1}{M_{\star}} \sum_{x \in S_{\star}^{1}, y \in S_{\star}^{2}} \operatorname{cap}_{S}(x, y) \sum_{\substack{\xi \in E_{N}-1 \\ \xi_{x}+\xi_{y} \geq N-\ell \\ \xi_{z} \leq b-1, \forall z \in S \backslash S_{\star}}} \frac{N^{\alpha}}{Z_{N, S}} \frac{m_{\star}^{\xi}}{a(\xi)}\left\{F\left(\xi+\mathfrak{d}_{x}\right)-F\left(\xi+\mathfrak{d}_{y}\right)\right\}^{2} .
$$

Fix $x_{0} \in S_{\star}^{1}, y_{0} \in S_{\star}^{2}$ and let $S_{0}:=S \backslash\left\{x_{0}, y_{0}\right\}$. For each $k \geq 0$, let $B_{k}=B_{k}^{x_{0}, y_{0}}$ be the set of configurations on $S_{0}$ given by

$$
B_{k}=\left\{\zeta \in \mathbb{N}^{S_{0}}: \sum_{v \in S_{0}} \zeta_{v}=k ; \zeta_{z} \leq b-1, z \in S \backslash S_{\star}\right\} .
$$

For $\zeta$ in $B_{k}$, let $G_{\zeta}:\{0, \ldots, N-1-k\} \rightarrow \mathbb{R}$ be defined as $G_{\zeta}(i)=F(\xi)$, where $\xi \in$ $E_{N-1}$ is the configuration given by $\xi_{v}=\zeta_{v}, v \in S_{0}, \xi_{x_{0}}=i$ and $\xi_{y_{0}}=N-1-k-i$. With this notation, for $x_{0} \in S_{\star}^{1}, y_{0} \in S_{\star}^{2}$ fixed, we may rewrite the second sum in the previous formula as

$$
\frac{N^{\alpha}}{Z_{N, S}} \sum_{k=0}^{\ell} \sum_{\zeta \in B_{k}} \frac{m_{\star}^{\zeta}}{a(\zeta)} \sum_{i=0}^{N-2-k} \frac{1}{a(i) a(N-1-k-i)}\left\{G_{\zeta}(i+1)-G_{\zeta}(i)\right\}^{2}
$$

because $m_{\star}\left(x_{0}\right)=m_{\star}\left(y_{0}\right)=1$. Note that $G_{\zeta}$ is equal to 0 on the set $\left\{0, \ldots, \ell_{N}-k\right\}$, and equal to 1 on the set $\left\{N-\ell_{N}, \ldots, N-1-k\right\}$. We may therefore restrict the 
sum over $i$ to a subset. It is easy to derive a lower bound for

$$
\sum_{i=\ell_{N}-k}^{N-\ell_{N}-1} \frac{1}{a(i) a(N-1-k-i)}\left\{G_{\zeta}(i+1)-G_{\zeta}(i)\right\}^{2} .
$$

The function $G$ which minimizes this expression is given by $G\left(N-\ell_{N}\right)=1$,

$$
G(i+1)-G(i)=\frac{1}{K_{N}} a(i) a(N-1-k-i), \quad i \in\left[\ell_{N}-k, N-\ell_{N}-1\right],
$$

where $K_{N}$ is a normalizing constant to ensure the boundary condition $G\left(\ell_{N}-k\right)=$ 0 . The respective lower bound is

$$
\Xi_{N}\left(x_{0}, y_{0}\right):=\left\{\sum_{i=\ell_{N}-k}^{N-\ell_{N}-1} a(i) a(N-1-k-i)\right\}^{-1} .
$$

This expression depends on the configuration $\zeta$ only through its number of particles. Moreover, for every fixed $k, N^{1+2 \alpha} \Xi_{N}\left(x_{0}, y_{0}\right)$ converges to $I_{\alpha}^{-1}$ as $N \uparrow \infty$.

In conclusion,

$$
N^{\alpha+1} D_{N}(F) \geq \frac{1}{M_{\star}} \sum_{x \in S_{\star}^{1}, y \in S_{\star}^{2}} \operatorname{cap}_{S}(x, y) \frac{N^{2 \alpha+1}}{Z_{N, S}} \sum_{k=0}^{\ell} \sum_{\zeta \in B_{k}} \Xi_{N}(x, y) \frac{m_{\star}^{\zeta}}{a(\zeta)} .
$$

By Proposition 2.1 and the above conclusions, as $N \uparrow \infty$, the right hand side converges to

$$
\frac{1}{M_{\star} I_{\alpha} Z_{S}} \sum_{x \in S_{\star}^{1}, y \in S_{\star}^{2}} \operatorname{cap}_{S}(x, y) \sum_{k=0}^{\ell} \sum_{\zeta \in B_{k}} \frac{m_{\star}^{\zeta}}{a(\zeta)} .
$$

Recall that $\ell$ and $b$ are free parameters introduced in the definition of the strip $L_{N}^{x, y}$. Thus, letting $b \uparrow \infty$ and then $\ell \uparrow \infty$, the second sum in the last expression converges to

$$
\sum_{k \geq 0} \sum_{\zeta \in E_{k, S \backslash\{x, y\}}} \frac{m_{\star}^{\zeta}}{a(\zeta)}=\prod_{z \in S \backslash\{x, y\}} \sum_{j \geq 0} \frac{m_{\star}(z)^{j}}{a(j)}=\prod_{z \in S \backslash\{x, y\}} \Gamma_{z}=\frac{Z_{S}}{\kappa_{\star} \Gamma(\alpha)} .
$$

For the last equation we have used the explicit formula of $Z_{S}$ presented just before Proposition 2.1. This proves the lemma.

\section{UPPER BOUND}

We prove in this section an upper bound for the capacity. As in the previous section, it might be simpler in a first reading to assume that $m$ is constant so that $S=S_{\star}$.

Proposition 5.1. Assume that $\kappa_{\star} \geq 2$. Fix a nonempty subset $S_{\star}^{1} \subsetneq S_{\star}$ and denote $S_{\star}^{2}=S_{\star} \backslash S_{\star}^{1}$. Then,

$$
\limsup _{N \rightarrow \infty} N^{1+\alpha} \operatorname{cap}_{N}\left(\mathcal{E}_{N}\left(S_{\star}^{1}\right), \mathcal{E}_{N}\left(S_{\star}^{2}\right)\right) \leq \frac{1}{M_{\star} \kappa_{\star} \Gamma(\alpha) I_{\alpha}} \sum_{x \in S_{\star}^{1}, y \in S_{\star}^{2}} \operatorname{cap}_{S}(x, y) .
$$

In view of the variational formula for the capacity, to obtain an upper bound for $\operatorname{cap}_{N}\left(\mathcal{E}_{N}\left(S_{\star}^{1}\right), \mathcal{E}_{N}\left(S_{\star}^{2}\right)\right)$, we need to choose a suitable function belonging to $\mathcal{C}_{N}\left(\mathcal{E}_{N}\left(S_{\star}^{1}\right), \mathcal{E}_{N}\left(S_{\star}^{2}\right)\right)$ and to compute its Dirichlet form. Recalling the proof of the lower bound, we expect this candidate to depend on the function which solves 
the variational problem for the capacity of the underlying random walk and on the optimal function for the zero range process with two sites.

To introduce the candidate, fix $x \in S_{\star}^{1}, y \in S_{\star}^{2}$ and recall the definition of the tube $L_{N}^{x, y}$. In view of the proof of the lower bound, the optimal function $F \in \mathcal{C}_{N}\left(\mathcal{E}_{N}\left(S_{\star}^{1}\right), \mathcal{E}_{N}\left(S_{\star}^{2}\right)\right)$ on the tube $L_{N}^{x, y}$ should satisfy

$$
\begin{aligned}
F\left(\xi+\mathfrak{d}_{w}\right)-F\left(\xi+\mathfrak{d}_{z}\right) & =\left\{\mathbf{f}_{\mathbf{x y}}(w)-\mathbf{f}_{\mathbf{x y}}(z)\right\}\left\{F\left(\xi+\mathfrak{d}_{x}\right)-F\left(\xi+\mathfrak{d}_{y}\right)\right\} \\
& =\left\{\mathbf{f}_{\mathbf{x y}}(w)-\mathbf{f}_{\mathbf{x y}}(z)\right\}\left\{G\left(\xi_{x}+1\right)-G\left(\xi_{x}\right)\right\},
\end{aligned}
$$

where $\mathbf{f}_{\mathbf{x}, \mathbf{y}}$ is the function which solves the variational problem $(2.3)$ in $\mathcal{B}(x, y)$ for the capacity of the underlying random walk, and $G$ is the function appearing in the proof of the lower bound.

Since, on the tube $L_{N}^{x, y}, \sum_{z \neq x, y} \xi_{z} \leq \ell_{N}$ and $G$ is a smooth function, paying a small cost we may replace $\xi_{x}$ in the previous formula by $\xi_{x}+\sum_{z \in A} \xi_{z}$ for any suitable set $A \subset S \backslash\{x, y\}$. The natural candidate on the strip $L_{N}^{x, y}$ is therefore

$$
\hat{F}_{x y}(\xi):=\sum_{j=1}^{\kappa-1}\left\{\mathbf{f}_{\mathbf{x y}}\left(z_{j}\right)-\mathbf{f}_{\mathbf{x y}}\left(z_{j+1}\right)\right\} G\left(\xi_{z_{1}}+\cdots+\xi_{z_{j}}\right)
$$

where $x=z_{1}, z_{2}, \ldots, z_{\kappa}=y$ is an enumeration of $S$ such that $\mathbf{f}_{\mathbf{x y}}\left(z_{j}\right) \geq \mathbf{f}_{\mathbf{x}, \mathbf{y}}\left(z_{j+1}\right)$ for $1 \leq j<\kappa$. A simple computation shows that this function has the required properties listed in the previous paragraph.

Since the tubes $L_{N}^{x, y}, x \in S_{\star}^{1}, y \in S_{\star}^{2}$, are essentially disjoints, the candidate $F$ should be equal to $\hat{F}_{x y}$ on each tube $L_{N}^{x, y}$ and equal to some appropriate convex combination of these functions on the complement.

We hope that this informal explanation helps to understand the rigorous and detailed definition of the candidate we now present. Let $\mathcal{D} \subset \mathbb{R}^{S}$ be the compact subset

$$
\mathcal{D}:=\left\{u \in \mathbb{R}_{+}^{S}: \sum_{x \in S} u_{x}=1\right\} .
$$

For each different sites $x, y \in S$ and $\delta>0$, consider the subsets of $\mathcal{D}$

$$
\mathcal{D}_{\delta}^{x}:=\left\{u \in \mathcal{D}: u_{x}>1-\delta\right\} \quad \text { and } \quad \mathcal{L}_{\delta}^{x y}:=\left\{u \in \mathcal{D}: u_{x}+u_{y} \geq 1-\delta\right\}
$$

Clearly $\mathcal{L}_{\delta}^{x y}=\mathcal{L}_{\delta}^{y x}$ for any $x, y \in S$.

Fix an arbitrary $0<\epsilon<1 / 6$ and $x$ in $S$. Let $\mathcal{K}_{y}^{x}=\mathcal{K}_{y}^{x}(\epsilon):=\mathcal{L}_{\epsilon}^{x y} \backslash \mathcal{D}_{3 \epsilon}^{x}, y \neq x$. Since $\mathcal{K}_{y}^{x}, y \in S \backslash\{x\}$, is a collection of pairwise disjoint compact subsets of $\mathcal{D}$, there is a family of smooth functions

$$
\Theta_{y}^{x}: \mathcal{D} \rightarrow[0,1], \quad y \in S \backslash\{x\},
$$

such that $\sum_{y \in S \backslash\{x\}} \Theta_{y}^{x}(u)=1$ for all $u$ in $\mathcal{D}$, and $\Theta_{y}^{x}(u)=1$ for all $u$ in $\mathcal{K}_{y}^{x}$ and $y \in S \backslash\{x\}$.

Clearly, the sets $\mathcal{L}_{\epsilon}^{x y}$ are macroscopic versions of the strips $L_{N}^{x, y}$. The functions $\Theta_{y}^{x}$ will be used to define the candidate function in the complement of the cylinders $L_{N}^{x, y}$.

Let $H:[0,1] \rightarrow[0,1]$ be the smooth function given by

$$
H(t):=\frac{1}{I_{\alpha}} \int_{0}^{\phi(t)} u^{\alpha}(1-u)^{\alpha} d u,
$$

where $I_{\alpha}$ is the constant defined in $(2.8)$ and $\phi:[0,1] \rightarrow[0,1]$ is a smooth bijective function such that $\phi(t)+\phi(1-t)=1$ for every $t \in[0,1]$ and $\phi(s)=0 \forall s \in[0,3 \epsilon]$. 
It can be easily checked that

$$
H(t)+H(1-t)=1, \quad \forall t \in[0,1],
$$

$\left.H\right|_{[0,3 \epsilon]} \equiv 0$ and $\left.H\right|_{[1-3 \epsilon, 1]} \equiv 1$. The function $H$ is a smooth approximation of the function $G$ which appeared in the proof of the lower bound.

Recall that $x \in S$ is fixed. For each $y \in S \backslash\{x\}$ consider the function $\mathbf{f}_{\mathbf{x y}}: S \rightarrow$ $[0,1]$ in $\mathcal{B}(x, y)$ such that

$$
D_{S}\left(\mathbf{f}_{\mathbf{x y}}\right)=\operatorname{cap}_{S}(x, y)=\inf _{f \in \mathcal{B}(x, y)} D_{S}(f) .
$$

It is well known that $\mathbf{f}_{\mathbf{x y}}(z)$ is equal to the probability that the random walk with generator $\mathcal{L}_{S}$ reaches $x$ before $y$ when it starts from $z$.

For each $y \in S \backslash\{x\}$ fix an enumeration

$$
x=z_{1}, z_{2}, \ldots, z_{\kappa}=y
$$

of $S$ satisfying $\mathbf{f}_{\mathbf{x y}}\left(z_{j}\right) \geq \mathbf{f}_{\mathbf{x y}}\left(z_{j+1}\right)$ for $1 \leq j \leq \kappa-1$ and define $F_{x y}: E_{N} \rightarrow \mathbb{R}$ as the convex linear combination

$$
F_{x y}(\eta):=\sum_{j=1}^{\kappa-1}\left\{\mathbf{f}_{\mathbf{x y}}\left(z_{j}\right)-\mathbf{f}_{\mathbf{x y}}\left(z_{j+1}\right)\right\} F_{x y}^{j}(\eta), \quad \eta \in E_{N},
$$

where each $F_{x y}^{j}: E_{N} \rightarrow \mathbb{R}, 1 \leq j \leq \kappa-1$, is given by $F_{x y}^{1}(\eta)=H\left(\eta_{x} / N\right)$ and

$$
F_{x y}^{j}(\eta):=H\left(\frac{\eta_{x}}{N}+\min \left\{\frac{1}{N} \sum_{i=2}^{j} \eta_{z_{i}} ; \epsilon\right\}\right), \quad \eta \in E_{N},
$$

for $2 \leq j \leq \kappa-1$.

The function $F_{x y}$ just defined is a smooth approximation of the function $\hat{F}_{x, y}$ defined at the beginning of this section. It is therefore the candidate to solve the variational problem for the capacity on the tube $L_{N}^{x, y}$. It remains to define $F_{x y}$ in the exterior of the cylinders.

Let $F_{x}: E_{N} \rightarrow \mathbb{R}$ be given by

$$
F_{x}(\eta):=\sum_{y \in S \backslash\{x\}} \Theta_{y}^{x}(\eta / N) F_{x y}(\eta),
$$

where each $\eta / N$ is thought of as a point in $\mathcal{D}$ and $\left\{\Theta_{y}^{x}: y \in S \backslash\{x\}\right\}$ is the partition of unity established before.

The following properties of $F_{x}$ are helpful in the proof of Proposition 5.1. It is easy to check that

$$
F_{x}(\eta)=F_{x y}(\eta) \text { for } \eta / N \in \mathcal{L}_{\epsilon}^{x y} .
$$

Indeed, if $\eta / N$ belongs to $\mathcal{K}_{y}^{x}, \Theta_{y}^{x}(\eta / N)=1$ proving the identity claimed. On the other hand, if $\eta / N$ belongs to $\mathcal{D}_{3 \epsilon}^{x}$, by definition of $H, F_{x z}^{j}(\eta)=1$ for all $z \in S \backslash\{x\}$, $1 \leq j \leq \kappa-1$, so that $F_{x z}(\eta)=F_{x}(\eta)$. By similar reasons,

$$
\begin{aligned}
F_{x} \equiv 1 \quad \text { on }\left\{\eta \in E_{N}: \eta_{x} \geq(1-3 \epsilon) N\right\} \\
\text { and } F_{x} \equiv 0 \quad \text { on }\left\{\eta \in E_{N}: \eta_{x} \leq 2 \epsilon N\right\} .
\end{aligned}
$$

The minimum in definition (5.3) is introduced precisely to fulfill the second assertion in (5.5). In particular, if $\eta / N \in \mathcal{D}_{2 \epsilon}^{z}$ for some $z \in S$ then

$$
F_{x}(\eta)=\mathbf{1}\{z=x\} .
$$


Since $H$, as well as each $\Theta_{y}^{x}$, is a smooth function, there exists a finite constant $C_{\epsilon}$, which depends on $\epsilon$ through the definition of the smooth functions, but does not depend on $N \geq 1$, such that

$$
\max _{\eta \in E_{N}}\left|F_{x}\left(\sigma^{z w} \eta\right)-F_{x}(\eta)\right| \leq \frac{C_{\epsilon}}{N}
$$

for every $z, w \in S$.

Let

$$
\mathcal{J}_{N}^{x y}:=\left\{\eta \in E_{N}: \eta_{x}+\eta_{y} \geq N-\ell_{N}\right\}, \quad x \neq y \in S .
$$

Clearly, $\mathcal{J}_{N}^{x y}=\mathcal{J}_{N}^{y x}, x, y \in S$ and, for every $N$ large enough, $\mathcal{J}_{N}^{x y} \subseteq \mathcal{L}_{\epsilon}^{x y}$. Let $\mathcal{J}_{N}^{x}:=\cup_{y \in S \backslash\{x\}} \mathcal{J}_{N}^{x y}$. In what follows, the value of the constant $C_{\epsilon}$ may change from line to line, but will never depend on $N$.

Lemma 5.2. For each $x \in S$ and every $N \geq 1$ large enough,

$$
\frac{1}{2} \sum_{\eta \in E_{N} \backslash \mathcal{J}_{N}^{x}} \sum_{z, w \in S} \mu_{N}(\eta) g\left(\eta_{z}\right) r(z, w)\left\{F_{x}\left(\sigma^{z w} \eta\right)-F_{x}(\eta)\right\}^{2} \leq \frac{C_{\epsilon} m_{\star}(x)^{\epsilon N}}{N^{\alpha+1}\left(\epsilon \ell_{N}\right)^{\alpha-1}} .
$$

Proof. By property (5.5), we can restrict the sum in the left hand side to configurations $\eta \in E_{N} \backslash \mathcal{J}_{N}^{x}$ satisfying $\epsilon N \leq \eta_{x} \leq(1-\epsilon) N$. So, by (5.7), the left hand side of the above inequality is bounded above by

$$
\frac{C_{\epsilon}}{N^{2}} \sum_{\substack{\eta \in E_{N} \backslash \mathcal{J}_{N}^{x} \\ \epsilon N \leq \eta_{x} \leq(1-\epsilon) N}} \mu_{N}(\eta)
$$

This expression is bounded above by

$$
\frac{N^{\alpha} C_{\epsilon}}{Z_{N, S} N^{2}} \sum_{\epsilon N \leq i \leq(1-\epsilon) N} \sum_{\substack{\eta: \eta_{x}=i \\ \max \left\{\eta_{y}: y \neq x\right\} \leq N-i-\ell_{N}}} \frac{m_{\star}^{\eta}}{a(\eta)}
$$

which can be re-written as

$$
\frac{N^{\alpha} C_{\epsilon}}{Z_{N, S} N^{2}} \sum_{\epsilon N \leq i \leq(1-\epsilon) N} \frac{m_{\star}(x)^{i}}{a(i) a(N-i)}\left\{(N-i)^{\alpha} \sum_{\zeta \in E_{N-i, S \backslash\{x\}}\left(\ell_{N}\right)} \frac{m_{\star}^{\zeta}}{a(\zeta)}\right\} .
$$

By Lemma 3.2 for the expression inside braces, last expression is bounded above by

$$
\frac{C_{\epsilon} m_{\star}(x)^{\epsilon N}}{Z_{N, S} N^{2} \ell_{N}^{\alpha-1}}\left\{N^{\alpha} \sum_{\epsilon N \leq i \leq(1-\epsilon) N} \frac{1}{a(i) a(N-i)}\right\} .
$$

By Lemma 3.2 once more and Proposition 2.1 we obtain the desired result.

Fix a nonempty subset $S^{1} \subsetneq S$ and denote $S^{2}:=S \backslash S^{1} \neq \varnothing$. We define the function $F_{S^{1}}: E_{N} \rightarrow \mathbb{R}$ as

$$
F_{S^{1}}(\eta):=\sum_{x \in S^{1}} F_{x}(\eta) .
$$

Let us define the following subsets of $E_{N}$

$$
D_{N}^{x}:=\left\{\eta \in E_{N}: \eta_{x} \geq N-3 \ell_{N}\right\}, \quad x \in S,
$$

so that $\mathcal{E}_{N}^{x} \subset D_{N}^{x}, x \in S_{\star}$. It follows from (5.5) that if $\eta \in D_{N}^{x}$ for some $x \in S$ then

$$
F_{S^{1}}(\eta)=\mathbf{1}\left\{x \in S^{1}\right\}=F_{S^{1}}\left(\sigma^{z w} \eta\right),
$$


for every $z, w \in S$ and every $N$ large enough. In particular,

$$
F_{S^{1}} \in \mathcal{C}_{N}\left(\bigcup_{x \in S^{1}} D_{N}^{x}, \bigcup_{y \in S^{2}} D_{N}^{y}\right)
$$

We shall use $F_{S^{1}}$ to get an upper bound for $\operatorname{cap}_{N}\left(\cup_{x \in S^{1}} D_{N}^{x}, \cup_{y \in S^{2}} D_{N}^{y}\right)$.

We first claim that for any $N$ large enough,

$$
F_{S^{1}}\left(\sigma^{z w} \eta\right)=1=F_{S^{1}}(\eta) \text { for all } \eta \in \bigcup_{x, y \in S^{1}} \mathcal{J}_{N}^{x y} \text { and } z, w \in S .
$$

To prove this claim, fix $x \neq y$ in $S^{1}$. By (5.5), (5.4), for $\eta / N \in \mathcal{L}_{\epsilon}^{x y}$,

$$
F_{S^{1}}(\eta)=F_{x y}(\eta)+F_{y x}(\eta) \text {. }
$$

Recall from (5.2) the enumeration of $S$ defined according to the values of $\mathbf{f}_{\mathbf{x y y}}$. Let $z_{1}, \ldots, z_{\kappa}$ and $w_{1}, \ldots, w_{\kappa}$ be such enumerations obtained from $\mathbf{f}_{\mathbf{x y}}$ and $\mathbf{f}_{\mathbf{y x}}$, respectively. Since $\mathbf{f}_{\mathbf{x y}}+\mathbf{f}_{\mathbf{y x}} \equiv 1$, we can choose the enumerations in such a way that $z_{n+1}=w_{\kappa-n}, 0 \leq n \leq \kappa-1$. With this convention, an elementary computation shows that

$$
F_{x y}(\eta)+F_{y x}(\eta)=\sum_{j=1}^{\kappa-1}\left\{\mathbf{f}_{\mathbf{x y}}\left(z_{j}\right)-\mathbf{f}_{\mathbf{x y}}\left(z_{j+1}\right)\right\}\left(F_{x y}^{j}(\eta)+F_{y x}^{\kappa-j}(\eta)\right) .
$$

By (5.1), the previous expression is equal to

$$
\sum_{j=1}^{\kappa-1}\left\{\mathbf{f}_{\mathbf{x y}}\left(z_{j}\right)-\mathbf{f}_{\mathbf{x y}}\left(z_{j+1}\right)\right\}=1 .
$$

Claim (5.9) follows from this identity and (5.10) since $\mathfrak{J}_{N}^{x y} \subset \mathcal{L}_{\epsilon}^{x y}$ for $N$ sufficiently large.

For each subset $A \subseteq E_{N}$ and function $F: E_{N} \rightarrow \mathbb{R}$, let

$$
D_{N}(F ; A):=\frac{1}{2} \sum_{\eta \in A} \sum_{z, w \in S} \mu_{N}(\eta) g\left(\eta_{z}\right) r(z, w)\left\{F\left(\sigma^{z w} \eta\right)-F(\eta)\right\}^{2} .
$$

With this notation, Lemma 5.2 can be stated as

$$
D_{N}\left(F_{x} ; E_{N} \backslash \mathcal{J}_{N}^{x}\right) \leq \frac{C_{\epsilon} m_{\star}(x)^{\epsilon N}}{N^{\alpha+1}\left(\epsilon \ell_{N}\right)^{\alpha-1}} \quad \forall x \in S .
$$

By Cauchy-Schwarz inequality,

$$
\begin{aligned}
D_{N}\left(F_{S^{1}} ; E_{N} \backslash \cup_{z \in S^{1}} \mathcal{J}_{N}^{z}\right) & \leq\left|S^{1}\right| \sum_{x \in S^{1}} D_{N}\left(F_{x} ; E_{N} \backslash \cup_{z \in S^{1}} \mathcal{J}_{N}^{z}\right) \\
& \leq\left|S^{1}\right| \sum_{x \in S^{1}} D_{N}\left(F_{x} ; E_{N} \backslash \mathcal{J}_{N}^{x}\right) .
\end{aligned}
$$

Therefore, since $\ell_{N} \uparrow \infty$, it follows from Lemma 5.2 that

$$
\lim _{N \rightarrow \infty} N^{\alpha+1} D_{N}\left(F_{S^{1}} ; E_{N} \backslash \cup_{z \in S^{1}} \mathrm{~J}_{N}^{z}\right)=0 .
$$

It remains to estimate $D_{N}\left(F_{S^{1}} ; \cup_{z \in S^{1}} \mathcal{J}_{N}^{z}\right)$. By definition of $\mathcal{J}_{N}^{z}, z \in S^{1}$, and by (5.9),

$$
D_{N}\left(F_{S^{1}} ; \bigcup_{z \in S^{1}} \mathcal{J}_{N}^{z}\right)=D_{N}\left(F_{S^{1}} ; \bigcup_{\substack{x \in S^{1} \\ y \in S^{2}}} \mathcal{J}_{N}^{x y}\right)=\sum_{x \in S^{1}} \sum_{y \in S^{2}} D_{N}\left(F_{S^{1}} ; \mathcal{J}_{N}^{x y}\right) .
$$


The last identity follows from (5.8) and the relation

$$
\mathcal{J}_{N}^{x_{1} y_{1}} \cap \mathcal{J}_{N}^{x_{2} y_{2}} \subseteq \bigcup_{z \in S} D_{N}^{z} \quad \text { for all } x_{1}, x_{2} \in S^{1} \text { and } y_{1}, y_{2} \in S^{2} .
$$

Therefore, by (5.5) and (5.4) we finally conclude that

$$
D_{N}\left(F_{S^{1}} ; \bigcup_{z \in S^{1}} \mathfrak{J}_{N}^{z}\right)=\sum_{x \in S^{1}} \sum_{y \in S^{2}} D_{N}\left(F_{x y} ; \mathfrak{J}_{N}^{x y}\right) .
$$

We now provide an estimate for each term in this sum. To derive this bound, in addition to the properties already imposed to the function $\phi$, we also require that

$$
\sup \left\{\phi^{\prime}(u): u \in[0,1]\right\} \leq 1+\sqrt{\epsilon}
$$

and

$$
\sup \left\{\frac{\phi(u)}{u-\epsilon}: u \in[2 \epsilon, 1]\right\} \leq 1+\sqrt{\epsilon} .
$$

The first requirement can easily be accomplished since $(1+\sqrt{\epsilon})$ times the length of the interval $[3 \epsilon, 1-3 \epsilon]$ is strictly greater than 1 for $\epsilon$ small enough. For (5.14), it suffices that $\phi(u) \leq(u-\epsilon)(1+\sqrt{\epsilon})$ for all $u \in[3 \epsilon, 1]$ because $\phi$ vanishes on $[0,3 \epsilon]$. Since $(u-\epsilon)(1+\sqrt{\epsilon})>1$ for $u=1-3 \epsilon$ and every $\epsilon$ small enough, it is possible to define a smooth function $\phi$ satisfying (5.14) without violating the other previously imposed properties.

According to the above discussion, in what follows we suppose that $\epsilon$ is an arbitrary number in $\left(0, \epsilon_{0}\right]$ for a suitably chosen $\epsilon_{0}>0$ and that $\phi$ satisfies the additional properties (5.13) and (5.14).

Proposition 5.3. For any $x, y \in S, x \neq y$,

$$
\limsup _{N \rightarrow \infty} N^{\alpha+1} D_{N}\left(F_{x y} ; \mathcal{J}_{N}^{x y}\right) \leq \frac{(1+\sqrt{\epsilon})^{2 \alpha+1}}{M_{\star} \kappa_{\star} I_{\alpha} \Gamma(\alpha)} \operatorname{cap}_{S}(x, y) \mathbf{1}\left\{x, y \in S_{\star}\right\} .
$$

Proof. Let $x=z_{1}, z_{2}, \ldots, z_{\kappa}=y$ be the enumeration established in the definition of $F_{x y}$, so that $\mathbf{f}_{\mathbf{x y}}\left(z_{n}\right) \geq \mathbf{f}_{\mathbf{x y}}\left(z_{n+1}\right), 1 \leq n \leq \kappa-1$. Fix two different sites $z_{i} \neq z_{j}$ in $S$ with $1 \leq i<j \leq \kappa$. By definition of $F_{x y}$,

$$
F_{x y}\left(\sigma^{z_{i} z_{j}} \eta\right)-F_{x y}(\eta)=\sum_{n=i}^{j-1}\left(\mathbf{f}_{\mathbf{x y}}\left(z_{n}\right)-\mathbf{f}_{\mathbf{x y}}\left(z_{n+1}\right)\right)\left\{F_{x y}^{n}\left(\sigma^{z_{i} z_{j}} \eta\right)-F_{x y}^{n}(\eta)\right\} .
$$

Thus, by the Cauchy-Schwarz inequality, the sum

$$
\sum_{\eta \in \mathcal{J}_{N}^{x y}} \mu_{N}(\eta) g\left(\eta_{z_{i}}\right) r\left(z_{i}, z_{j}\right)\left\{F_{x y}\left(\sigma^{z_{i} z_{j}} \eta\right)-F_{x y}(\eta)\right\}^{2}
$$

is bounded above by $\left\{\mathbf{f}_{\mathbf{x y}}\left(z_{i}\right)-\mathbf{f}_{\mathbf{x y}}\left(z_{j}\right)\right\}$ times

$$
\sum_{n=i}^{j-1}\left(\mathbf{f}_{\mathbf{x y}}\left(z_{n}\right)-\mathbf{f}_{\mathbf{x y}}\left(z_{n+1}\right)\right) \sum_{\eta \in \mathcal{J}_{N}^{x y}} \mu_{N}(\eta) g\left(\eta_{z_{i}}\right) r\left(z_{i}, z_{j}\right)\left\{F_{x y}^{n}\left(\sigma^{z_{i} z_{j}} \eta\right)-F_{x y}^{n}(\eta)\right\}^{2} .
$$

Performing the change of variables $\xi=\eta-\mathfrak{d}_{z_{i}}$, the second sum above is less than

$$
m_{\star}\left(z_{i}\right) r\left(z_{i}, z_{j}\right) \frac{N^{\alpha}}{Z_{N, S}} \sum_{\xi \in A_{N}^{x y}} \frac{m_{\star}^{\xi}}{a(\xi)}\left\{F_{x y}^{n}(\xi)-F_{x y}^{n}\left(\xi+\mathfrak{d}_{z_{i}}\right)\right\}^{2},
$$


where $A_{N}^{x y}:=\left\{\xi \in E_{N-1, S}: \xi_{x}+\xi_{y} \geq N-2 \ell_{N}\right\}$. So far, we have shown that (5.15) is bounded above by $m_{\star}\left(z_{i}\right) r\left(z_{i}, z_{j}\right) N^{\alpha} Z_{N, S}^{-1}\left\{\mathbf{f}_{\mathbf{x y}}\left(z_{i}\right)-\mathbf{f}_{\mathbf{x y}}\left(z_{j}\right)\right\}$ times

$$
\sum_{n=i}^{j-1}\left(\mathbf{f}_{\mathbf{x y}}\left(z_{n}\right)-\mathbf{f}_{\mathbf{x y}}\left(z_{n+1}\right)\right) \sum_{\xi \in A_{N}^{x y}} \frac{m_{\star}^{\xi}}{a(\xi)}\left\{H\left(\frac{1}{N}+\sum_{r=1}^{n} \frac{\xi_{z_{r}}}{N}\right)-H\left(\sum_{r=1}^{n} \frac{\xi_{z_{r}}}{N}\right)\right\}^{2} .
$$

Fix some $i \leq n<j$. The second sum in the above expression may be re-written as

$$
\begin{array}{r}
\sum_{m=0}^{2 \ell_{N}} \sum_{\zeta \in E_{m, S \backslash\{x, y\}}} \frac{m_{\star}^{\zeta}}{a(\zeta)} \sum_{\epsilon N \leq k \leq(1-2 \epsilon) N} \frac{m_{\star}(x)^{k} m_{\star}(y)^{N-m-k}}{a(k) a(N-m-k)} \\
\left\{H\left(d_{k+1}(\zeta)\right)-H\left(d_{k}(\zeta)\right)\right\}^{2},
\end{array}
$$

where

$$
d_{k}(\zeta):=\frac{k}{N}+\mathbf{1}\{n \geq 2\} \sum_{r=2}^{n} \frac{\zeta_{z_{r}}}{N}, \quad \epsilon N \leq k \leq(1-2 \epsilon) N .
$$

To keep notation simple let $\phi_{k}$ stand for $\phi\left(d_{k}(\zeta)\right)$. By the Cauchy-Schwarz inequality, the last expression is less than $\left\{m_{\star}(x) m_{\star}(y)\right\}{ }^{\epsilon N} N^{-2 \alpha} I_{\alpha}^{-2}$ times

$$
\sum_{m=0}^{2 \ell_{N}} \sum_{\zeta \in E_{m, S \backslash\{x, y\}}} \frac{m_{\star}^{\zeta}}{a(\zeta)} \sum_{k=\epsilon N}^{(1-2 \epsilon) N} \int_{\phi_{k}}^{\phi_{k+1}} u^{\alpha}(1-u)^{\alpha} d u \int_{\phi_{k}}^{\phi_{k+1}} \frac{u^{\alpha}(1-u)^{\alpha}}{\left(\frac{k}{N}\right)^{\alpha}\left(1-\frac{k+m}{N}\right)^{\alpha}} d u .
$$

Since $m \leq 2 \ell_{N}$ then, for all $N$ large enough, the last integral above is less than

$$
\left\{\phi_{k+1}-\phi_{k}\right\}\left(\sup _{u \in[0,1]}\{\phi(u) /(u-\epsilon)\}\right)^{2 \alpha} \leq \frac{1}{N}(1+\sqrt{\epsilon})^{2 \alpha+1} .
$$

The last inequality follows from assumptions (5.13) and (5.14). Therefore, we conclude that (5.16) is bounded above by

$$
\frac{\left\{m_{\star}(x) m_{\star}(y)\right\}^{\epsilon N}(1+\sqrt{\epsilon})^{2 \alpha+1}}{I_{\alpha} N^{2 \alpha+1}} \sum_{m=0}^{2 \ell_{N}} \sum_{\zeta \in E_{m, S \backslash\{x, y\}}} \frac{m_{\star}^{\zeta}}{a(\zeta)},
$$

which in turn is bounded by

$$
\frac{Z_{S} \Gamma(\alpha)\left\{m_{\star}(x) m_{\star}(y)\right\}^{\epsilon N}(1+\sqrt{\epsilon})^{2 \alpha+1}}{\kappa_{\star} \Gamma_{x} \Gamma_{y} I_{\alpha} N^{2 \alpha+1}} .
$$

Hence, we have shown that (5.15) is bounded above by

$m\left(z_{i}\right) r\left(z_{i}, z_{j}\right)\left\{\mathbf{f}_{\mathbf{x y}}\left(z_{i}\right)-\mathbf{f}_{\mathbf{x y}}\left(z_{j}\right)\right\}^{2}\left(\frac{Z_{S} \Gamma(\alpha)\{1+\sqrt{\epsilon}\}^{2 \alpha+1}}{M_{\star} \kappa_{\star} Z_{N, S} \Gamma_{x} \Gamma_{y} I_{\alpha}}\right)\left\{m_{\star}(x) m_{\star}(y)\right\}^{\epsilon N} N^{-\alpha-1}$.

In a similar way we can get the same upper bound for (5.15) if we suppose instead that $j<i$. The assertion of the proposition follows from this estimate and Proposition 2.1.

We are now in a position to prove Proposition 5.1. Let $S_{\star}^{1}:=S^{1} \cap S_{\star}, S_{\star}^{2}:=$ $S^{2} \cap S_{\star}$ and suppose they are both nonempty sets. Since

$$
\operatorname{cap}_{N}\left(\mathcal{E}_{N}\left(S_{\star}^{1}\right), \mathcal{E}_{N}\left(S_{\star}^{2}\right)\right) \leq \operatorname{cap}_{N}\left(\bigcup_{x \in S^{1}} D_{N}^{x}, \bigcup_{y \in S^{2}} D_{N}^{y}\right) \leq D_{N}\left(F_{S^{1}}\right),
$$


it follows from (5.11), (5.12) and Proposition 5.3 that

$$
\limsup _{N \rightarrow \infty} N^{\alpha+1} \operatorname{cap}_{N}\left(\varepsilon_{N}\left(S_{\star}^{1}\right), \varepsilon_{N}\left(S_{\star}^{2}\right)\right) \leq \frac{1}{M_{\star} \kappa_{\star} I_{\alpha} \Gamma(\alpha)} \sum_{x \in S_{\star}^{1}, y \in S_{\star}^{2}} \operatorname{cap}_{S}(x, y),
$$

after letting $\epsilon \downarrow$ 0. Theorem 2.2 follows from Proposition 5.1 and Proposition 4.1.

\section{Proof of Theorem 2.4}

In [2], we reduced the proof of the metastability of reversible processes to the verification of three conditions, denoted by (H0), (H1) and (H2). The proof of condition (H1) is similar to the one presented in [3] for zero range processes on complete graphs. However, in the case where $m$ is not uniform, some modifications are needed to handle sites not in $S_{\star}$. This is the only reason for which we have introduced the sequences $b_{N}(z), z \in S \backslash S_{\star}$ and the respective condition in (2.10).

The following notation will be used throughout this section. For each $x \in S_{\star}$, let $\xi_{N}^{x} \in E_{N}$ be the configuration with $N$ particles at $x$ and let $\breve{\varepsilon}_{N}^{x}$ represent the set $\mathcal{E}_{N}\left(S_{\star} \backslash\{x\}\right)$.

Condition (H2) follows immediately from (3.2) since $\mu_{N}\left(\mathcal{E}_{N}^{x}\right) \rightarrow 1 / \kappa_{\star}$ for every $x \in S_{\star}$ :

$$
\lim _{N \rightarrow \infty} \frac{\mu_{N}\left(\Delta_{N}\right)}{\mu_{N}\left(\mathcal{E}_{N}^{x}\right)}=0, \quad \forall x \in S_{\star} .
$$

Fix a configuration $\eta$ in $\mathcal{E}_{N}^{x}$. Since $\sum_{y \neq x} \eta_{y} \leq \ell_{N}$ and $\eta_{z} \leq b_{N}$, for $z \in S \backslash S_{\star}$, by the explicit form of $\mu_{N}$,

$$
\begin{aligned}
\mu_{N}(\eta) & \geq C_{0} \prod_{z \in S \backslash S_{\star}} m_{\star}(z)^{\eta_{z}} \prod_{y \in S \backslash\{x\}} \frac{1}{a\left(\eta_{y}\right)} \\
& \geq \frac{C_{0}}{\ell_{N}^{\alpha(\kappa-1)}} \prod_{z \in S \backslash S_{\star}} m_{\star}(z)^{b_{N}(z)} .
\end{aligned}
$$

Hereafter, $C_{0}$ stands for a constant which does not depend on $N \geq 1$ and whose value may change from line to line. To estimate the capacity, $\operatorname{cap}_{N}\left(\{\eta\},\left\{\xi_{N}^{x}\right\}\right)$ we consider a path $\eta^{(j)}, 0 \leq j \leq p$, from $\eta^{(0)}=\eta$ to $\eta^{(p)}=\xi_{N}^{x}$ obtained by moving to $x$, one by one, each particle. Since there are at most $\ell_{N}$ particles to move, we can take a path such that $p \leq \kappa \ell_{N}$. Let $F$ be an arbitrary function in $\mathcal{C}_{N}\left(\{\eta\},\left\{\xi_{N}^{x}\right\}\right)$. By Cauchy-Schwarz inequality and the explicit expression of the Dirichlet form,

$$
1=\left\{\sum_{j=0}^{p-1}\left[F\left(\eta^{(j+1)}\right)-F\left(\eta^{(j)}\right)\right]\right\}^{2} \leq C_{0} D_{N}(F) \sum_{j=0}^{p-1} \frac{1}{\mu_{N}\left(\eta^{(j)}\right)} .
$$

Therefore, by (6.1),

$$
\operatorname{cap}_{N}\left(\{\eta\},\left\{\xi_{N}^{x}\right\}\right) \geq \frac{C_{0}}{\ell_{N}^{1+\alpha(\kappa-1)}} \prod_{z \in S \backslash S_{\star}} m_{\star}(z)^{b_{N}(z)} .
$$

The extra factor $\ell_{N}$ comes from the length of the path. Condition (H1) follows now from this estimate, Theorem 2.2 and condition (2.10):

$$
\lim _{N \rightarrow \infty} \frac{\operatorname{cap}_{N}\left(\mathcal{E}_{N}^{x}, \breve{\varepsilon}_{N}^{x}\right)}{\inf _{\eta \in \mathcal{E}_{N}^{x}}\left\{\operatorname{cap}_{N}\left(\{\eta\},\left\{\xi_{N}^{x}\right\}\right)\right\}}=0, \quad \forall x \in S_{\star} .
$$


Finally condition (H0) follows from Theorem 2.2 as we show below. Denote by $R_{N}^{\mathcal{E}^{\star}}(\cdot, \cdot)$ the jump rates of the trace process $\left\{\eta_{t}^{\varepsilon_{N}^{\star}}: t \geq 0\right\}$ defined in Section 2. For $x \neq y$ in $S_{\star}$, let

$$
r_{N}(x, y):=\frac{1}{\mu_{N}\left(\mathcal{E}_{N}^{x}\right)} \sum_{\substack{\eta \in \mathcal{E}_{N}^{x} \\ \xi \in \mathcal{E}_{N}^{y}}} \mu_{N}(\eta) R_{N}^{\mathcal{E}^{\star}}(\eta, \xi) .
$$

By Lemma 6.8 in [2],

$$
\begin{aligned}
\mu_{N}\left(\mathcal{E}_{N}^{x}\right) r_{N}\left(\mathcal{E}_{N}^{x}, \mathcal{E}_{N}^{y}\right)=\frac{1}{2}\left\{\operatorname{cap}_{N}\left(\mathcal{E}_{N}^{x}, \breve{\mathcal{E}}_{N}^{x}\right)+\operatorname{cap}_{N}\left(\mathcal{E}_{N}^{y}, \breve{\mathcal{E}}_{N}^{y}\right)\right. \\
\left.-\operatorname{cap}_{N}\left(\mathcal{E}_{N}(\{x, y\}), \mathcal{E}_{N}\left(S_{\star} \backslash\{x, y\}\right)\right)\right\} .
\end{aligned}
$$

Therefore, by Theorem 2.2 , since $\mu_{N}\left(\mathcal{E}_{N}^{x}\right)$ converges to $\kappa_{\star}^{-1}$ for all $x$ in $S_{\star}$,

$$
\lim _{N \rightarrow \infty} N^{1+\alpha} r_{N}\left(\mathcal{E}_{N}^{x}, \mathcal{E}_{N}^{y}\right)=\frac{\operatorname{cap}_{S}(x, y)}{M_{\star} \Gamma(\alpha) M_{\alpha}}, \quad \forall x, y \in S_{\star}, x \neq y .
$$

This proves Theorem 2.4 as a consequence of Theorem 2.10 in [2].

\section{REFERENCES}

[1] I. Armendáriz, M. Loulakis. Thermodynamic limit for the invariant measures in supercritical zero range processes. Probab. Theory Related Fields 145, 175-188 (2009).

[2] J. Beltrán, C. Landim: Tunneling and metastability of continuous time Markov chains. preprint (2009).

[3] J. Beltrán, C. Landim: Tunneling and metastability of continuous time Markov chains II. preprint (2009).

[4] M. R. Evans, T. Hanney: Nonequilibrium statistical mechanics of the zero-range process and related models. J. Phys. A 38(19), R195-R240 (2005)

[5] M. R. Evans, S. N. Majumdar, R. K. P. Zia: Canonical analysis of condensation in factorised steady states. J. Stat. Phys. 123, 357-390 (2006)

[6] P. A. Ferrari, C. Landim, V. V. Sisko. Condensation for a fixed number of independent random variables. J. Stat. Phys. 128, 1153-1158 (2007).

[7] C. Godrèche, J. M. Luck: Dynamics of the condensate in zero-range processes. J. Phys. A 38, 7215-7237 (2005)

[8] S. Großkinsky, G. M. Schütz, H. Spohn. Condensation in the zero range process: stationary and dynamical properties. J. Statist. Phys. 113, 389-410 (2003)

[9] I. Jeon, P. March, B. Pittel: Size of the largest cluster under zero-range invariant measures. Ann. Probab. 28, 1162-1194 (2000)

Institut de Mathématiques, École Polytechnique Fédérale de Lausanne, Station 8 , CH-1015 Lausanne, Switzerland. and PUCP, Av. Universitaria cdra. 18, San Miguel, AP. 1761, Lima 100, Perú.

E-MAIL: johel@impa.br

impa, Estrada Dona Castorina 110, CeP 22460 Rio de Janeiro, Brasil and CNRS UMR 6085, Université de Rouen, Avenue de l'Université, BP.12, Technopôle du MadrilLet, F76801 Saint-Étienne-du-Rouvray, France.

E-MAIL: landim@impa.br 